山्山FRANÇAISE

$>\mathrm{DE}$

$\stackrel{1=1}{\simeq}$ PÉDAGOGIE
Revue française de pédagogie

Recherches en éducation

$195 \mid 2016$

Varia

\title{
Fréquenter des pairs très performants n'a pas que des vertus : impact de l'école ou de la classe fréquentée sur le concept de soi scolaire (le BFLPE)
}

Being assigned to highly performing groups is not always beneficial: impact of the class or school attended on academic self-concept

Virginie Dupont et Dominique Lafontaine

\section{OpenEdition}

Journals

Édition électronique

URL : http://journals.openedition.org/rfp/5037

DOI : $10.4000 /$ rfp.5037

ISSN : 2105-2913

Éditeur

ENS Éditions

Édition imprimée

Date de publication : 30 juin 2016

Pagination : 63-86

ISSN : 0556-7807

Référence électronique

Virginie Dupont et Dominique Lafontaine, « Fréquenter des pairs très performants n'a pas que des vertus : impact de l'école ou de la classe fréquentée sur le concept de soi scolaire (le BFLPE) », Revue française de pédagogie [En ligne], 195 | 2016, mis en ligne le 30 juin 2019, consulté le 04 janvier 2021. URL : http://journals.openedition.org/rfp/5037 ; DOI : https://doi.org/10.4000/rfp.5037 


\section{Fréquenter des pairs très performants n'a pas que des vertus: impact de l'école ou de la classe fréquentée sur le concept de soi scolaire (le BFLPE)}

Virginie Dupont

Dominique Lafontaine

Cette note de synthèse porte sur un phénomène connu sous le nom de «Big-Fish-LittlePond Effect» (BFLPE; l'effet «gros poisson dans le petit étang»). Les études consacrées à cet effet montrent que la fréquentation d'une école ou d'une classe aux performances moyennes élevées peut ne pas être favorable sur le plan socio-affectif. Plus précisément, il apparaît qu'à performances individuelles équivalentes, fréquenter une école ou une classe aux performances élevées est associé à un concept de soi académique plus faible. Cet effet dû aux comparaisons sociales réalisées avec le groupe de référence que constitue l'école ou la classe est par ailleurs présent dans une large majorité de pays et touche l'ensemble des élèves quelles que soient leurs caractéristiques.

Mots-clés (TESE) : confiance en soi, école, classe, environnement, système éducatif. 


\section{Introduction}

Depuis la naissance des courants de la School Effectiveness Research et du School Mix, nombre d'études portant sur les effets écoles ont mis en évidence l'importance du contexte scolaire fréquenté sur les acquis cognitifs et socio-affectifs. On tend généralement à considérer que fréquenter une école performante ou socialement favorisée est d'emblée associé à des conséquences positives pour le jeune tant sur le plan des performances (Duru-Bellat, 2003; Monseur \& Lafontaine, 2009), de ses aspirations scolaires (Felouzis, 2003; Duru-Bellat, Danner, Le Bastard-Landrier et al., 2004; Nakhili, 2005) que sur le plan motivationnel (Caslyn \& Kenny, 1977; Müller \& Hofmann, 2014). Pourtant, les choses ne sont pas si simples. Marsh et ses collaborateurs ont en effet publié de nombreux travaux portant sur le concept de soi académique (perception de soi dans le cadre scolaire) et ont mis en évidence la présence d'un «Big-Fish-Little-Pond Effect » (BFLPE) : le poisson qui nage dans un petit étang se sent plus gros que s'il nageait dans l'océan. Traduit dans le contexte scolaire, cela signifie que fréquenter une école aux performances moyennes élevées peut être préjudiciable pour le concept de soi académique de l'élève. C'est ce phénomène qui sera étudié de manière approfondie dans cette note de synthèse.

Depuis 1984, de nombreuses études ont été réalisées sur le BFLPE, pour la plupart par Marsh et ses collaborateurs. C'est principalement sur la base de cette abondante littérature que la présente note de synthèse a été rédigée. L'ensemble des études répertoriées ont la particularité d'envisager les performances des élèves à partir de tests standardisés et font, dans la grande majorité des cas, appel à des analyses multiniveaux. Par contre, elles se distinguent sur deux aspects principaux : I'origine des bases de données (internationale ou nationale) et le niveau envisagé pour calculer les performances moyennes du groupe de référence (école ou classe). Les études peuvent ainsi être classées en quatre catégories : les études basées sur des données internationales et se situant au niveau école, les études basées sur des données internationales au niveau classe, les études basées sur des données nationales au niveau école et enfin les études basées sur des données nationales de niveau classe. L'analyse de cette catégorisation (voir annexe 1) permet de dégager plusieurs constats. Tout d'abord, la majorité des études réalisées avec des données internationales sont au niveau école. Ces études sont en effet presque toutes réalisées au départ des données de l'enquête internationale Program for International Student Assessment (PISA) qui échantillonne aléatoirement des élèves au sein des écoles et non des classes entières. À l'inverse, les études réalisées au niveau classe l'ont davantage été sur la base de données nationales. Ensuite, il est à noter que les études basées sur des données nationales au niveau école reprennent notamment les études de première génération réalisées sur le BFLPE, c'est-à-dire avant les années 2000. Enfin, si les premières études menées sur le BFLPE I'ont principalement été au départ de données australiennes, par la suite, les recherches au niveau national ont été réalisées majoritairement en Allemagne. L'analyse de l'ensemble de ces articles permet de dégager plusieurs grandes thématiques qui seront abordées dans cette note de synthèse.

Dans un premier temps, nous nous attacherons à définir le BFLPE en remontant à ses origines et en définissant les concepts au cœur de cet effet. Nous tenterons ainsi de comprendre le lien entre les performances moyennes de l'école fréquentée et le concept de soi académique des élèves. Comme nous le verrons, les comparaisons sociales jouent un rôle majeur dans la compréhension de ce phénomène. À un niveau plus méthodologique, nous reviendrons sur l'importance de tester cet effet en mesurant les performances des élèves au départ de tests standardisés (identiques pour tous) et sur le rôle des notes scolaires dans ce contexte.

Dans un deuxième temps, nous tenterons de voir comment le BFLPE s'inscrit dans les théories de la comparaison sociale. Selon la forme qu'elles prennent, les comparaisons sociales 
peuvent engendrer à la fois un effet négatif et un effet positif sur les évaluations de soi. Nous verrons dans quelle mesure ces deux types de comparaisons peuvent coexister.

Dans un troisième temps, la note s'attardera sur le caractère universel, généralisable, persistant et étendu du BFLPE. Marsh et ses collaborateurs ont en effet souligné le caractère robuste de ce phénomène. Tout d'abord, le caractère universel du BFLPE sera questionné. Par universel nous entendons le fait que cet effet soit observé dans une très grande majorité de pays. Afin de pouvoir comparer les résultats entre pays, ces recherches se sont centrées sur les bases de données internationales telles que le Program for International Student Assessment (PISA) et Trends in International Mathematics and Science Study (TIMSS). Ensuite, nous nous centrerons sur le caractère généralisable du BFLPE, c'est-à-dire la mesure dans laquelle cet effet concerne tous les élèves, quelles que soient leurs caractéristiques tant socio-démographiques, scolaires, qu'attitudinales. La persistance du BFLPE sera également étudiée. S'agit-il d'un effet à court ou à long terme? Les performances moyennes de l'école que l'élève fréquente aujourd'hui peuvent-elles avoir un impact sur l'image qu'il aura de lui dans le futur? Enfin, nous envisagerons la possibilité que le BFLPE ne se limite pas au concept de soi académique, mais qu'il puisse également toucher d'autres aspects tels que les aspirations ou l'intérêt.

Dans un quatrième temps, nous envisagerons les différents cadres de référence pris en compte dans les études sur le BFLPE. Certaines études opérationnalisent le cadre de référence au travers des performances moyennes de l'école alors que d'autres prennent en compte les performances moyennes de la classe ou de la filière. Quel est, en fin de compte, le niveau le plus adéquat pour étudier cet effet?

Enfin, nous terminerons cette note de synthèse en discutant les résultats des recherches à la lumière des caractéristiques de celles-ci. Travailler au départ de données nationales ou internationales, se situer au niveau classe ou au niveau école conduit-il à des résultats différents? À quels enseignements ces différents types de méthodologies conduisent-ils? Nous discuterons également des implications que ces résultats peuvent avoir sur la pratique et envisagerons les pistes de recherches pour le futur.

\section{Origine du BFLPE et clarification conceptuelle}

En 1984, Marsh et Parker, à partir de données de 305 élèves australiens du grade 6 fréquentant cinq écoles différentes, étudient le lien entre les habiletés scolaires, les performances moyennes de l'école et le concept de soi académique de l'élève, selon le modèle présenté à la figure 1. Les habiletés scolaires des élèves sont ici mesurées grâce à deux tests standardisés : un test de quotient intellectuel et un test en lecture, tandis que la performance de l'école est établie en réalisant la moyenne des habiletés scolaires des élèves de l'école. Ils mettent ainsi en évidence que, si les performances de l'élève sont positivement liées à son concept de soi académique, à compétences égales, celui-ci est influencé négativement par les performances moyennes de l'école fréquentée. Autrement dit, si l'on compare des élèves aux performances similaires, mais dont certains fréquentent une école performante et d'autres une école moins performante, ceux qui fréquentent cette dernière ont un concept de soi académique plus favorable que ceux qui fréquentent l'école plus performante. Le BFLPE est mis en évidence.

$\mathrm{Si}$, à l'origine, l'effet négatif des performances moyennes de l'école a été observé pour le concept de soi académique, par la suite il a également été mis en évidence pour le concept de soi relatif à une matière en particulier (mathématiques, sciences, français). Par contre, le BFLPE n'est pas présent lorsqu'on prend en considération un concept de soi plus général (Marsh \& Parker, 1984; Marsh, 1987; Marsh, Chessor, Craven et al., 1995). Le concept de soi fait référence aux perceptions de soi construites au travers des expériences faites avec son environnement (Pietsch, Walker \& Chapman, 2003). C'est un concept multidimensionnel dans le sens où il peut être différent selon l'objet sur lequel il porte (l'école, le sport, l'apparence physique...). Ainsi, 


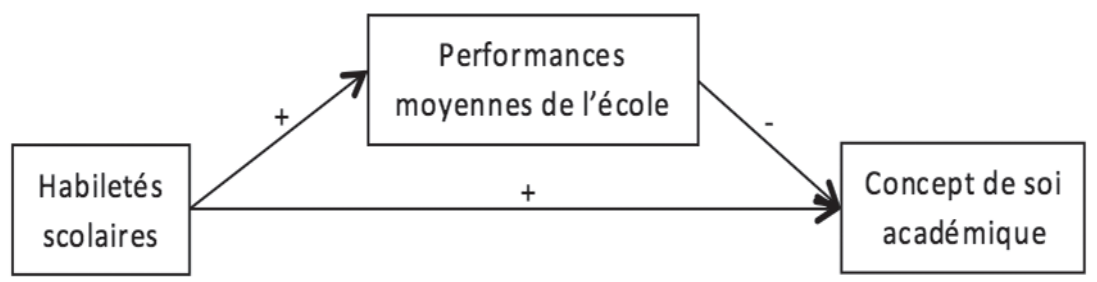

le concept de soi académique (ASC, «academic self-concept»), à la différence du concept de soi général, renvoie à la manière dont l'élève se perçoit dans le cadre scolaire ou dans une discipline scolaire donnée. Il est souvent mesuré à l'aide d'items tels que «je suis fort en maths », "je suis un des meilleurs de ma classe»... Ceci suppose une comparaison au moins implicite par rapport à d'autres élèves de la classe ou de l'école que l'on fréquente. On comprend immédiatement en quoi le concept de soi peut être influencé par la composition des groupes dans lesquels l'élève s'inscrit. Selon que ce groupe classe ou école est plus ou moins performant, selon que ce groupe est plus ou moins hétérogène, le point de comparaison que prend l'élève est variable et le concept de soi en est inévitablement affecté.

Ainsi, dès les premières études, il apparaît que le BFLPE est lié aux théories de la comparaison sociale. Les comparaisons sociales peuvent être considérées comme « un processus dans lequel les gens s'engagent de manière quasi automatique [...] et qu'ils emploient dans des buts divers : se rassurer quant à leurs opinions ou performances, évaluer ce qui est possible, gonfler leur estime de soi, se vacciner contre l'influence... Plus fondamentalement, la comparaison avec les autres permet à chacun de se définir comme sujet à la fois unique et semblable à autrui » (Leyens \& Yzerbyt, 1997, p. 27). Dans le cas du BFLPE, l'élève évalue ses propres performances scolaires (concept de soi académique) en les comparant aux performances de ses pairs, l'école constitue donc son cadre de référence. Selon le BFLPE, ces comparaisons ont un effet négatif sur le concept de soi académique des élèves qui fréquentent une école aux performances moyennes élevées. Cet effet négatif porte également le nom d'effet de contraste. Les comparaisons en jeu dans l'effet de contraste peuvent être considérées comme des comparaisons forcées car elles sont imposées par le cadre de référence (ici, l'école ou la classe).

Comme dans la recherche menée par Marsh et Parker en 1984, les recherches étudiant le BFLPE mesurent toujours les performances des élèves au départ de tests standardisés, c'està-dire de tests pour lesquels le contenu, les conditions de passation et de correction sont identiques pour tous. Cela est nécessaire puisque c'est la seule manière d'avoir des mesures comparables d'une classe ou d'une école à l'autre. Pourtant, il apparaît dans la recherche en éducation que les notes scolaires données par l'enseignant sont de meilleurs prédicteurs du concept de soi académique (Hansford \& Hattie, 1982; Skaalvik \& Skaalvik, 2002) que les performances obtenues à des tests standardisés. Ces notes scolaires ont toutefois rarement été prises en compte dans l'étude du BFLPE car, dans la majorité des cas, celles-ci sont établies sur la base d'une référence normative : les enseignants notent leurs élèves en les comparant les uns aux autres et reproduisent ainsi une courbe de Gauss (un peu d'élèves faibles, beaucoup d'élèves moyens et un peu d'élèves forts), c'est ce qu'on appelle l'effet Posthumus (ou gradingon-a-curve, en anglais). Si ces notes scolaires ne sont pas adéquates pour tester le BFLPE, il n'empêche que celles-ci donnent à l'élève un feed-back clair, accessible, fiable et pertinent sur 
ses aptitudes scolaires ainsi que sur sa position au sein de la classe ou de l'école. Ces informations sont donc susceptibles de renforcer l'effet de contraste. Marsh (1987), Trautwein, Lüdtke, Marsh et alii (2006), Marsh, Trautwein, Lüdtke et alii (2007) et Preckel et Brüll (2010) ont ainsi testé l'hypothèse selon laquelle l'effet des performances moyennes de la classe ou de l'école fréquentée sur le concept de soi académique serait médié par les notes scolaires données par I'enseignant. Ils confirment tous cette hypothèse : le BFLPE est en partie médié par les notes scolaires. Cela signifie que le BFLPE s'explique, en partie, par le caractère normatif des notes scolaires données par l'enseignant. À notes scolaires équivalentes, l'effet des performances moyennes de la classe sur le concept de soi reste toutefois significativement négatif. Cette significativité montre que le BFLPE ne s'explique pas complètement par ces notes scolaires.

\section{BFLPE et comparaisons sociales}

L'effet de contraste mis en évidence par Marsh et ses collaborateurs dans l'étude du BFLPE n'est pas la seule conséquence possible des comparaisons sociales en milieu scolaire. D'une part, les études menées en psychologie sociale et portant sur les comparaisons sociales en milieu scolaire (Blanton, Buunk, Gibbons et al., 1999; Huguet, Dumas, Monteil et al., 2001) mettent en évidence que se comparer, de façon libre, à des pairs plus performants que soi peut être inspirant plutôt que menaçant. D'autre part, comme l'ont signalé Trautwein, Lüdtke, Marsh et alii (2009), les élèves fréquentant une école performante pourraient avoir un meilleur concept de soi académique en raison de la fierté qu'ils ont d'appartenir à une école ou un programme d'étude d'élite (reflected glory effect). Dans les deux cas, cet effet positif est également appelé effet d'assimilation puisque l'individu cherche les similitudes entre lui et le sujet ou le groupe auquel il se compare afin de s'identifier à celui-ci. Comment BFLPE et reflected glory effect se combinent-ils? C'est à cette question que cette section va tenter de répondre.

\section{BFLPE et comparaisons sociales libres}

Selon Seaton, Marsh, Dumas et alii (2008), Marsh, Trautwein, Lüdtke et alii (2008) ou encore Huguet, Dumas, Marsh et alii (2009), c'est la source des comparaisons sociales qui diffère dans les études sur le BFLPE et celles sur les comparaisons sociales menées en psychologie sociale. Alors que les comparaisons mises en jeu dans le BFLPE sont forcées et implicites, c'est-à-dire que les élèves n'ont pas conscience qu'ils se comparent avec l'ensemble d'élèves auquel ils appartiennent, les comparaisons à l'œuvre dans les études telles que celles menées par Blanton, Buunk, Gibbons et alii (1999) ou encore Huguet, Dumas, Monteil et alii (2001) sont des comparaisons initiées par l'élève et donc explicites et libres. Seaton, Marsh, Dumas et alii (2008) ont mené de nouvelles analyses sur les données utilisées par Blanton, Buunk, Gibbons et alii (1999) et Huguet, Dumas, Monteil et alii (2001). L'objectif poursuivi par ces auteurs était de mettre en évidence, d'une part l'existence d'un BFLPE pour ces données et, d'autre part, la possible coexistence d'un effet positif des comparaisons ascendantes libres et d'un effet négatif des performances moyennes de l'école ou de la classe sur le concept de soi académique (BFLPE). La réanalyse des données de Blanton, Buunk, Gibbons et alii (1999), données récoltées auprès de 879 élèves du grade 9 aux Pays-Bas, montre que si les performances de l'individu influencent positivement son concept de soi, à compétences égales, les performances moyennes de la classe ont une influence négative sur ce concept de soi (BFLPE). Ils mettent également en évidence que, lorsqu'on prend en compte les comparaisons libres ascendantes (mesurées en demandant à l'élève à qui il compare ses notes en général) dans l'analyse, le BFLPE ne diminue pas. Par contre, ils observent que les comparaisons libres ascendantes n'ont pas d'effet direct sur le concept de soi. Chanal et Sarrazin (2007) font le même constat pour le concept de soi en éducation physique. La réanalyse des données de Huguet, Dumas, Monteil et alii (2001), portant 
sur 1156 élèves issus de 51 classes de 12 écoles françaises, permet quant à elle d'observer que l'effet positif des comparaisons libres avec quelqu'un de plus performant ne modère pas non plus le BFLPE. Dans la même lignée, Huguet, Dumas, Marsh et alii (2009), au départ de données françaises (2015 élèves de première année du secondaire issus de 99 classes de 16 écoles), montrent que choisir de se comparer à quelqu'un de plus performant que soi est associé à un meilleur concept de soi tant en mathématiques qu'en français. De plus, lorsqu'on contrôle l'effet des comparaisons libres sur le concept de soi, c'est-à-dire lorsqu'on dissocie l'effet propre des comparaisons libres et forcées, l'ampleur de l'effet négatif des performances moyennes de l'école augmente. Ainsi, Seaton, Marsh, Dumas et alii (2008) tout comme Huguet, Dumas, Monteil et alii (2001), Huguet, Dumas, Marsh et alii (2009) et Chanal et Sarrazin (2007) soulignent la coexistence et la relative indépendance du BFLPE et des comparaisons ascendantes libres.

\section{BFLPE et reflected glory effect}

Contrairement à ce qui est observé dans le cas du BFLPE, fréquenter une école aux performances moyennes élevées ou encore une filière plus sélective pourrait aussi être valorisant pour le jeune et affecter positivement son concept de soi. Appartenir à un groupe que l'on perçoit comme «bon » pourrait en effet être valorisant. Cet effet positif lié au groupe que l'on fréquente s'appelle le reflected glory effect ou effet d'assimilation. Deux approches distinctes ont été utilisées afin de mettre en évidence un possible effet d'assimilation lié à la fréquentation d'une école ou d'une filière plus prestigieuse. Soit les chercheurs essaient de capter l'effet d'assimilation en incluant dans l'analyse la filière fréquentée par l'élève (Marsh, Köller \& Baumert, 2001 ; Lüdtke, Köller, Marsh et al., 2005; Trautwein, Lüdtke, Marsh et al., 2006; Preckel \& Brüll, 2010), soit ils mesurent directement la perception par le jeune de la position, en termes de prestige, de l'école ou de la filière fréquentée (Marsh, Kong \& Hau, 2000; Trautwein, Lüdtke, Marsh et al., 2009). Dans les deux cas, l'objectif est de distinguer l'effet de contraste et l'effet d'assimilation confondus lorsque l'on envisage uniquement l'effet des performances moyennes de l'école sur le concept de soi.

À partir de données allemandes récoltées auprès 3787 élèves du grade 7 lors de l'année scolaire 1991-1992, Marsh, Köller et Baumert (2001) observent que, lorsqu'on inclut dans l'analyse à la fois les performances moyennes de la classe et le type de filière (Gymnasium ou non), l'effet négatif des performances moyennes de la classe est encore plus négatif que lorsque seules ces dernières sont incluses dans l'analyse; le type de filière a quant à lui un effet positif sur le concept de soi. Cet effet positif représente clairement l'effet d'assimilation lié au fait de fréquenter la filière la plus prestigieuse (Gymnasium). À l'inverse, Lüdtke, Köller, Marsh et alii (2005) qui ont réalisé le même type d'analyse au départ de données allemandes pour des élèves des grades 7 et 8 n'observent aucun effet de la filière fréquentée. En 2006, Trautwein, Lüdtke, Marsh et alii poussent plus loin l'analyse de l'effet d'assimilation au travers de la fréquentation des filières. Toujours au départ de données allemandes, les auteurs différencient les filières entre écoles et les «filières » au sein de l'école. Le caractère très différencié du système scolaire allemand permet en effet de réaliser cette distinction. Une majorité des Länder allemands se caractérisent par l'organisation de trois filières distinctes correspondant chaque fois à une école (Hauptschule, Realschule et Gymnasium) et une minorité comportent des écoles secondaires dites « compréhensives» (Gesamtschulen) au sein desquelles les élèves sont assignés dans des classes différentes selon leur niveau' pour les cours de mathématiques, de langues étrangères et d'allemand. On comprend aisément que, selon la modalité de filiarisation en vigueur, la manière dont un élève se construit un concept de soi peut varier sensiblement. C'est, entre autres, à cette ques- 
tion précise que cette étude sophistiquée s'intéresse. Les auteurs ont ainsi mené des analyses distinctes selon ces deux formes d'organisation de filières ou de classes de niveau. Dans le cas où les filières sont organisées entre écoles, Trautwein, Lüdtke, Marsh et alii (2006) observent bien un effet de contraste lié aux performances moyennes de l'école fréquentée. De manière plus surprenante, ils observent également un effet de contraste lié à la filière fréquentée, c'està-dire que le concept de soi en mathématiques d'élèves aux performances similaires fréquentant la filière Gymnasium (la plus prestigieuse) est plus faible que celui des élèves des autres filières. Autrement dit, même si les filières sont bien visibles, tant pour les élèves que pour les parents, la fréquentation de la filière la plus prestigieuse n'est pas associée à un effet positif au niveau du concept de soi en mathématiques. Dans le cas des classes de niveau organisées au sein des écoles, les résultats sont tout à fait différents. En effet, les auteurs constatent un effet de contraste lié aux performances moyennes de l'établissement et un effet d'assimilation lié à la fréquentation de la classe de niveau le plus avancé. Les auteurs mettent toutefois en évidence qu'il ne s'agit pas d'un réel effet d'assimilation. L'effet positif lié à la classe de niveau fréquentée s'explique en réalité par les notes que l'enseignant donne aux élèves : sous contrôle des notes scolaires, la classe de niveau n'a plus d'effet positif significatif sur le concept de soi académique. Ce résultat peut s'expliquer par le fait que les élèves qui fréquentent la «meilleure» classe reçoivent les meilleures notes de leurs enseignants (les enseignants ne recréent pas une courbe de Gauss pour chacune des classes de niveau), leur permettant ainsi de se sentir davantage aptes que les élèves des classes de niveau moins exigeantes.

Dans le même ordre d'idées, Preckel et Brüll (2010) étudient l'effet de contraste et l'effet d'assimilation au départ de données allemandes récoltées auprès d'élèves du grade 5 . Ces élèves fréquentent tous le Gymnasium, mais certains sont dans des classes dites « régulières » tandis que d'autres sont dans des classes où seuls les plus «doués » sont rassemblés. Les auteurs constatent un effet négatif significatif des performances moyennes de la classe uniquement lorsqu'on compare des classes équivalentes, le type de classe fréquentée ayant un effet positif sur le concept de soi en mathématiques. Ils constatent également, contrairement à Trautwein, Lüdtke, Marsh et alii (2006), que ces effets restent significatifs même quand les notes scolaires sont tenues sous contrôle. Même si ces résultats mettent en évidence la coexistence d'un effet de contraste et d'un effet d'assimilation, ils n'en restent pas moins surprenants puisque, à l'inverse de toutes les autres études, ils ne mettent pas clairement en évidence la prédominance du BFLPE. II est toutefois à noter que ces auteurs ont mesuré les performances des élèves à partir de tests de quotient intellectuel (QI). Ce QI pourrait être plus éloigné des performances scolaires que ne le sont les performances mesurées au départ de tests standardisés. De plus, l'effet d'assimilation particulièrement fort observé dans cette étude pourrait, selon les auteurs, s'expliquer par le fait que fréquenter des classes de niveau est nouveau pour les élèves (le grade 5 est la première année où les élèves sont répartis en filières et classes de niveau) et que cela exacerberait la fierté d'être dans une classe "prestigieuse».

Marsh, Kong et Hau (2000) ont étudié l'effet d'assimilation au travers du prestige perçu par l'élève de son école. Ils ont mené une étude auprès de 7997 élèves de grade 7 à HongKong, où il n'y a pas de filière d'enseignement, mais où les écoles diffèrent en termes de prestige et où être admis dans une école prestigieuse est très valorisé, parents et enfants étant bien conscients de la position des écoles les unes par rapport aux autres. Les résultats de cette étude montrent qu'à performances individuelles égales, les performances moyennes de l'école ont un effet négatif sur le concept de soi académique. Lorsqu'on inclut dans l'analyse la perception du prestige de l'école, celle-ci a un effet positif sur le concept de soi et, sous contrôle de cette variable, l'effet négatif des performances moyennes de l'école augmente en amplitude. Ces résultats attestent donc que fréquenter une école aux performances moyennes élevées implique à la fois des comparaisons «exigeantes» avec les autres élèves de l'école et peut engendrer un sentiment de fierté. 
Au départ de trois bases de données allemandes, Trautwein, Lüdtke, Marsh et alii (2009) testent également la possible coexistence d'un effet de contraste, mesuré grâce aux performances moyennes de la classe ou de l'école, et d'un effet d'assimilation, appréhendé au travers du prestige perçu par l'élève de sa classe ou de son école. Dans les trois études, menées au grade 13, les auteurs constatent qu'à compétences de l'élève comparables, il existe bien un effet négatif des performances moyennes de la classe ou de l'école sur le concept de soi académique; toutefois, ce dernier est également influencé par les perceptions qu'a l'élève du prestige relatif de sa classe ou de son école. Il semble ainsi que prendre en compte le prestige perçu de l'école ou de la classe soit une meilleure méthode pour mettre en évidence l'effet d'assimilation que la filière fréquentée, les résultats étant plus consistants dans ce cas.

Au final, le BFLPE au sens technique du terme (coefficient de régression négatif des performances moyennes de l'école sur le concept de soi) apparaît comme étant l'effet net ou la résultante d'influences de sens opposés : un fort effet de contraste (effet négatif) engendré par des comparaisons forcées et un effet d'assimilation (effet positif) plus faible engendré soit par des comparaisons libres avec quelqu'un de plus performant, soit par l'effet d'appartenance à un groupe positivement valorisé. Comme l'effet de contraste est généralement plus fort que l'effet d'assimilation, dans la majorité des cas, un BFLPE est observé.

\section{Le caractère robuste du BFLPE}

\section{Un BFLPE universel...}

Les premières études portant sur le BFLPE ont utilisé des bases de données nationales essentiellement en Australie (Marsh \& Parker, 1984; Marsh, 1987) et aux États-Unis (Marsh, 1991). Si les résultats issus de ces différentes recherches vont toujours dans le même sens et attestent un effet négatif des performances moyennes de l'école ou de la classe sur le concept de soi, il existe néanmoins des problèmes de comparabilité des résultats. En effet, pour pouvoir affirmer le caractère universel du BFLPE, c'est-à-dire sa présence dans tous les pays, il est nécessaire de disposer de données provenant de différents pays, mais récoltées sur la base d'échantillons comparables et portant sur des mesures identiques. Plusieurs recherches ont ainsi été menées au départ des données issues de PISA (Marsh \& Hau, 2003; Seaton, Marsh \& Craven, 2009; Nagengast \& Marsh, 2012) et de TIMSS (Marsh, Abduljabbar, Morin et al., 2015; Wang, 2015). La première (Marsh \& Hau, 2003) a été menée sur les données PISA 2000 et inclut vingt-six pays de I'OCDE. Cette étude met en évidence qu'à performances individuelles égales, le concept de soi académique est négativement influencé par les performances moyennes de l'école dans les vingt-six pays. Il est toutefois à noter que l'ampleur du lien varie de -0,02 à -0,36 et est non significatif dans deux des pays étudiés (Hongrie et Corée). Seaton, Marsh et Craven (2009) confirment cette tendance au départ des données PISA 2003, pour quarante et un pays. Dans tous les pays étudiés, ils constatent, en effet, qu'à performances égales, le concept de soi en mathématiques de l'élève est influencé négativement par les performances moyennes de l'école, ce lien étant toutefois non significatif dans trois pays (Islande, Irlande et Corée). De plus, ces auteurs tentent de voir si le caractère individualiste ou collectiviste de la culture du pays ainsi que son degré de développement économique (pays développé versus pays en voie de développement) permettent d'expliquer des différences d'ampleur du BFLPE d'un pays à l'autre. Les résultats montrent que l'effet négatif des performances moyennes de l'école est très légèrement supérieur dans les pays individualistes. Cependant, la différence est tellement petite que les auteurs affirment que le BFLPE est généralisable tant aux pays individualistes que collectivistes. En ce qui concerne le degré de développement économique du pays, aucune différence significative n'est à noter, l'effet négatif des performances moyennes de l'école sur le concept de soi étant similaire dans tous les pays, qu'ils soient en voie de développement 
ou développés. Enfin, sur la base des données PISA 2006, Nagengast et Marsh (2012) mettent en évidence un BFLPE dans cinquante des cinquante-six pays étudiés. Dans les six pays restants, l'effet des performances moyennes de l'école sur le concept de soi en sciences est non significatif. De plus, comme Seaton, Marsh et Craven (2009), les auteurs montrent que les effets des performances moyennes de l'école ne sont pas dépendants du niveau de développement économique du pays ni de son caractère collectiviste ou individualiste. Ces trois études permettent donc non seulement d'affirmer l'universalité du BFLPE, mais aussi son caractère généralisable à différents domaines scolaires (mathématiques et sciences).

Des études plus récentes ont été réalisées au départ des données de l'enquête TIMSS 2007. Marsh, Abduljabbar, Morin et alii (2015) étudient le BFLPE dans treize pays à partir des données pour les grades 4 et 8 . Ici, le cadre de référence correspond à la classe et non plus à l'école comme c'était le cas avec les données PISA. Les auteurs constatent un BFLPE significatif dans les treize pays étudiés, tant au grade 4 qu'au grade 8 . Ils notent toutefois des différences significatives en fonction du grade et du pays étudié. Ainsi, ils mettent en évidence que l'effet négatif des performances moyennes de l'école est de plus grande ampleur au secondaire qu'au primaire. De plus, selon la classification des auteurs, le BFLPE semble également de plus grande ampleur dans les pays de culture occidentale (Australie, Angleterre, Italie, Norvège, Écosse, États-Unis) et en Asie (Hong-Kong, Japon, Taïwan, Singapour) que dans les pays islamiques du Moyen-Orient ${ }^{2}$ (Iran, Koweït, Tunisie) présents dans l'étude. Wang (2015) trouve lui aussi un effet négatif significatif des performances moyennes de la classe fréquentée au grade 8 sur le concept de soi en mathématiques dans les quarante-neuf pays qu'il a étudiés au départ des données TIMSS 2007.

Au travers de ces différentes études, le caractère universel du BFLPE semble donc s'affirmer. En effet, quel que soit le pays étudié, les élèves aux performances similaires ont un concept de soi académique, mathématique ou encore scientifique plus faible lorsqu'ils fréquentent une école ou une classe aux performances moyennes élevées. Certaines études (Marsh, Köller \& Baumert, 2001; Nagengast \& Marsh, 2011; Van de Gaer, Grisay, Schulz et al., 2012) invitent toutefois à nuancer ce propos : le BFLPE serait d'autant plus important que le système scolaire dans lequel il est observé est peu inclusif. En effet, dans les systèmes scolaires compréhensifs, c'est-à-dire où les élèves ne sont pas regroupés dans des classes ou des écoles de niveaux différents, les cadres de référence sur la base desquels les élèves s'auto-évaluent sont relativement homogènes. À l'inverse, dans les systèmes différenciés, où les élèves sont regroupés par niveau, les cadres de référence sont différents d'une classe ou d'une école à l'autre, ce qui décuple les effets des comparaisons sociales.

Sur la question du lien entre le BFLPE et l'organisation des systèmes éducatifs, une étude déjà ancienne menée par Marsh, Köller et Baumert (2001) en Allemagne apporte un éclairage unique. Au moment de sa réunification, I'Allemagne constitue un terrain particulièrement intéressant pour étudier l'impact du caractère différencié du système éducatif. En effet, alors que les élèves d'Allemagne de l'Ouest sont scolarisés dans un système comprenant différentes filières avant la réunification, ceux d'Allemagne de l'Est sont, quant à eux, habitués à un système non différencié. Au moment de la réunification, c'est le système différencié, déjà en application à I'Ouest, qui a été étendu en Allemagne de l'Est. Ainsi, des données ont été recueillies auprès d'élèves du grade 7 en début, milieu et fin d'année scolaire 1991-1992. Les auteurs font l'hypothèse que les élèves d'Allemagne de l'Est, qui n'ont donc pas connu de système scolaire différencié jusque-là, seront moins affectés par le BFLPE que ceux d'Allemagne de l'Ouest. L'hypothèse est pleinement confirmée : au moment de la réunification, l'effet des performances moyennes de l'école est significativement plus négatif pour les élèves d'Allemagne de l'Ouest que pour 
ceux d'Allemagne de l'Est. Les auteurs notent également que cette différence est toujours significative, mais de moindre ampleur en milieu d'année et qu'elle n'est plus significative en fin d'année. À ce moment, tous les élèves se sont accoutumés au système différencié qui est désormais leur lot commun. Dans un tel système, le concept de soi des élèves subit davantage l'influence des performances moyennes de leur classe que dans un modèle intégré.

Dans le même ordre d'idées, Nagengast et Marsh (2011) ont réalisé une étude au départ des données PISA 2006 pour le Royaume-Uni. Les auteurs font le constat que l'effet négatif des performances moyennes de l'école sur le concept de soi en Irlande du Nord et en Angleterre est de plus forte ampleur qu'en Écosse et au Pays de Galles. Nagengast et Marsh (2011) interprètent ce résultat à la lumière du caractère plus ou moins différencié du système scolaire de chaque pays : I'Irlande du Nord est le seul pays du Royaume-Uni à avoir un système scolaire différencié avant l'âge de quinze ans. En Angleterre, il existe des écoles publiques sélectives mais leur nombre est assez limité, la plupart des écoles secondaires étant compréhensives. Enfin, au Pays de Galles et en Écosse, toutes les écoles sont compréhensives.

Toujours au départ des données PISA 2006 et à l'aide d'analyses multiniveaux (élèves, écoles, pays), Van de Gaer, Grisay, Schulz et alii (2012) ont également exploré l'existence d'un effet du groupe de référence. Ils constatent la présence d'un BFLPE : à compétences en sciences égales dans le test PISA, les élèves qui fréquentent une école plus performante ont un moins bon concept de soi en sciences. Cet effet négatif de la moyenne des performances de l'école sur le concept de soi est d'autant plus marqué que le pays compte une proportion élevée d'écoles sélectives (forte variance ou ségrégation entre écoles), autrement dit quand le système est fortement différencié.

Si l'intensité du BFLPE varie en fonction du caractère plus ou moins intégré du système éducatif, cela ne remet toutefois pas en cause l'universalité du BFLPE. Celui-ci a, en effet, été observé dans la grande majorité des systèmes éducatifs étudiés.

\section{Généralisable...}

Comme les recherches évoquées ci-dessus l'attestent, le caractère universel du BFLPE n'est plus à démontrer. Nous pouvons maintenant nous interroger sur la mesure dans laquelle cet effet est généralisable à tous les élèves. Certaines catégories d'élèves ne sont-elles pas davantage sensibles à la comparaison avec des pairs très performants? Dans cette partie, plusieurs caractéristiques de l'élève seront envisagées comme variables modératrices du BFLPE : le niveau d'aptitude de l'élève, des variables d'ordre socio-démographique telles que le sexe et le statut socio-économique, les attitudes scolaires ou encore certains traits de personnalité. Nous y envisagerons également certaines caractéristiques et pratiques pédagogiques de classe qui pourraient agir comme modérateurs du BFLPE.

\section{Le niveau d'aptitude de l'élève comme modérateur du BFLPE}

Selon Marsh et O'Mara (2010), a priori, le BFLPE devrait concerner tous les élèves. En effet, quelles que soient ses performances individuelles, chaque élève est confronté au cadre de référence que constitue son école ou sa classe. Ainsi, Marsh et ses collaborateurs (Marsh, 1987, 1991; Marsh \& Craven, 2002; Marsh, Seaton, Trautwein et al., 2008) indiquent que les effets d'interactions entre les performances moyennes de la classe ou de l'école et le niveau d'aptitude de l'élève sur le concept de soi académique devraient être faibles ou non significatifs puisque le cadre de référence est établi sur la base des performances moyennes de la classe ou de l'école.

Même si ces effets d'interaction sont faibles, un certain nombre de recherches mettent toutefois en évidence un BFLPE d'ampleur différente selon le niveau d'aptitude de l'élève. Les résultats ne vont toutefois pas systématiquement dans le même sens. Seaton, Marsh et Craven (2010) ont montré au départ des données PISA 2003 (tous pays confondus) que, même si le 
BFLPE concerne tous les élèves quel que soit leur niveau académique, l'effet négatif des performances moyennes de l'école est légèrement plus fort pour les élèves les plus performants. Marsh et Hau (2003) établissent le même constat en testant ce même effet d'interaction sur un ensemble de vingt-six pays issus de la base de données PISA 2000. Toujours au départ des données PISA, mais de 2006 cette fois, Nagengast et Marsh (2012) étudient les effets d'interaction pays par pays. Ils constatent ainsi que dans quarante-deux des cinquante-six pays étudiés les effets d'interaction sont significatifs : l'effet négatif des performances moyennes de l'école serait plus prononcé pour les élèves forts. L'effet d'interaction est non significatif dans les quatorze autres pays. À l'inverse, Seaton, Marsh, Dumas et alii (2008), Trautwein, Lüdtke, Marsh et alii (2009) ou encore Huguet, Dumas, Marsh et alii (2009) mettent en évidence la présence d'un BFLPE pour tous les élèves, mais de moindre ampleur pour les élèves les plus performants. Autrement dit, le concept de soi académique des élèves les plus performants souffre moins de l'effet négatif de fréquenter une école très performante que celui des élèves faibles. Il est cependant à noter que ces résultats concernent spécifiquement des données françaises (base de données PISA et nationale) et allemandes (base de données nationale). Dans d'autres pays comme l'Australie, par exemple, où les données PISA 2000, 2003 et 2006 ont été analysées, on ne retrouve pas cet effet d'interaction, le BFLPE étant similaire quel que soit le niveau académique de l'élève (Seaton, Marsh, Yeung et al., 2011). En 1995, Marsh, Chessor, Craven et alii ont également constaté une absence d'interaction entre le niveau d'aptitude de l'élève et les performances moyennes de l'école au départ de données nationales australiennes. Contrairement à Trautwein, Lüdtke, Marsh et alii (2009), Marsh, Köller et Baumert (2001) et Marsh, Trautwein, Lüdkte et alii (2007), à l'aide de données nationales, constatent également une absence d'effet modérateur des aptitudes des élèves en Allemagne.

On voit, au travers de ces études, toute la complexité de trancher ou non pour un effet modérateur du niveau d'aptitude de l'élève. Marsh et ses collaborateurs soulignent quant à eux le caractère généralisable du BFLPE quel que soit le niveau académique de l'élève, en raison de l'absence de résultats consistants permettant d'affirmer le contraire. En effet, même s'il existe de légères variations dans l'intensité de l'effet négatif des performances moyennes sur le concept de soi académique, dans toutes les études nous sommes bien en présence d'un BFLPE. Une analyse un peu plus poussée des résultats présentés ci-dessus, en fonction de la base de données utilisée et du niveau d'analyse (classe ou école), ne permet pas de mettre en évidence des tendances significatives : les bases des données nationales mettent aussi bien en évidence des résultats non significatifs (Allemagne, Pays-Bas, France) qu'un BFLPE plus fort pour les élèves faibles (Allemagne, France) ou encore l'inverse (France). L'analyse des bases de données PISA met quant à elle en évidence un BFLPE plus prononcé pour les élèves les plus forts lorsque les analyses sont menées au travers de tous les pays et des résultats non significatifs ou un BFLPE plus prononcé pour les plus forts lorsque les données des pays sont analysées distinctement. L'analyse selon le niveau classe ou école ne permet pas non plus de dégager de tendances nettes.

\section{Les variables socio-démographiques de l'élève comme modérateurs du BFLPE}

Les quelques études ayant envisagé les variables socio-démographiques comme modérateurs du BFLPE ont pris en compte le sexe et l'origine socio-économique.

En ce qui concerne le sexe, si, en 1995, Marsh, Chessor, Craven et alii ont observé que l'ampleur du BFLPE ne varie pas en fonction du genre, d'autres études plus récentes n'arrivent pas à la même conclusion. Marsh, Trautwein, Lüdtke et alii (2007) ainsi que Plieninger et Dickhäuser (2015) ont en effet observé que le BFLPE peut varier en fonction du genre : à compétences individuelles équivalentes, l'effet négatif des performances moyennes de l'école sur le concept de soi académique de l'individu est plus marqué pour les filles. 
Au départ de deux études menées en Allemagne, Marsh, Trautwein, Lüdtke et alii (2007) ont étudié la possibilité que le BFLPE diffère selon le sexe du jeune. Ainsi, ils observent un effet négatif des performances moyennes de l'école d'ampleur plus grande pour les filles dans la première étude tandis qu'aucune différence significative au niveau du BFLPE n'est observée en fonction du genre dans la deuxième. Les auteurs concluent donc à un effet marginal. De leur côté, Plieninger et Dickhäuser (2015), au départ des données PISA 2006, ont montré que les filles semblent être plus affectées que les garçons par les performances de leurs camarades de classe. À performances égales en sciences, les filles souffrent davantage que les garçons de l'effet négatif des performances moyennes de leur école. Plieninger et Dickhäuser (2015) expliquent ce résultat de deux manières : d'une part, les filles s'engagent plus fréquemment dans des comparaisons sociales que les garçons, d'autre part, les filles rapportent un plus haut degré d'attachement à leurs pairs. Le fait que les mesures de Plieninger et Dickhäuser portent sur les sciences pourrait également expliquer ce résultat puisque c'est un domaine dans lequel les filles se sentent moins performantes, à compétences égales (Gavray \& Adriaenssens, 2010).

En ce qui concerne l'origine socio-économique, à notre connaissance, Seaton, Marsh et Craven (2010) sont les seuls à avoir étudié l'effet modérateur de l'origine socio-économique sur le BFLPE. Au départ des données PISA 2003, ils appréhendent le statut socio-économique de l'élève à partir de quatre éléments distincts : la profession des parents, le niveau de diplôme des parents, les ressources éducatives à la maison et les possessions culturelles. Ils constatent que seule l'interaction entre les performances moyennes de l'école et les possessions culturelles est significative. Toutefois, l'ampleur de celle-ci est tellement faible que les auteurs la considèrent comme trop petite pour devoir être discutée.

\section{Les attitudes scolaires comme variable modératrice du BFLPE}

Seaton, Marsh et Craven (2010) ont étudié une série d'attitudes scolaires comme potentiels modérateurs du BFLPE au départ des données PISA 2003 (à travers quarante et un pays). Sans surprise, ils constatent qu'à performances individuelles égales, l'effet négatif des performances moyennes de l'école sur le concept de soi est toujours présent; ils relèvent toutefois certaines conditions dans lesquelles celui-ci est accentué de manière significative. Ils constatent entre autres que si le sentiment de compétences, le sentiment d'appartenance, les relations avec l'enseignant, la motivation intrinsèque, la motivation extrinsèque et les attitudes compétitives ne modèrent pas le BFLPE, l'anxiété en mathématiques et les attitudes coopératives exacerbent celui-ci. Ainsi, les élèves qui ont un niveau d'anxiété en mathématiques plus faible sont moins touchés par l'effet négatif des performances moyennes de l'école, à l'inverse des élèves très anxieux qui souffrent davantage de cet effet. Les auteurs notent également que plus les élèves se montrent coopératifs et plus leur concept de soi en mathématiques pâtit de l'effet négatif de fréquenter une école aux performances élevées.

En 2011, Seaton, Marsh, Yeung et alii ont réalisé une étude similaire à celle de Seaton, Marsh et Craven (2010). Ils ont étudié l'effet modérateur des mêmes attitudes scolaires que ces derniers au départ des données PISA 2000, 2003 et 2006, mais uniquement pour l'Australie. II apparaît dans cette recherche qu'aucun des modérateurs envisagés n'agit comme tel. L'effet négatif des performances moyennes est ici similaire quelles que soient les méthodes d'apprentissage, la motivation ou les attitudes.

Enfin, Wouters, Colpin, Van Damme et alii (2015) ont étudié les buts d'apprentissage comme possibles modérateurs du BFLPE. Ils distinguent trois types de buts : les buts de maîtrise ${ }^{3}$, les buts de performance-approche et les buts de performance-évitement ${ }^{4}$. Au départ d'une étude

3 «Dans les buts de maîtrise, la préoccupation principale de la personne est d'augmenter ses savoirs et savoirfaire, d'améliorer sa compréhension et se maîtrise d'une tâche» (Galand \& Bourgeois, 2006, p. 65).

4 "Les buts de performance-approche, sous-tendus par un souci de valorisation de soi, s'expriment par la pré- 
menée en Flandre portant sur des élèves de 174 classes du grade 6 de 122 écoles, les auteurs constatent que les différents types de buts modèrent le BFLPE. Celui-ci est, en effet, exacerbé tant pour les étudiants qui poursuivent davantage des buts de performance-approche que pour ceux poursuivant des buts de performance-évitement et des buts de maîtrise. Si ce résultat peut sembler surprenant de prime abord, les auteurs expliquent qu'en ce qui concerne les buts de performances, il n'en est rien étant donné que ceux-ci sont directement liés aux comparaisons sociales (souci d'être meilleur que les autres, peur d'être moins bon que les autres). En ce qui concerne le résultat observé pour les buts de maîtrise, il est en lien avec de récents résultats de recherches qui montrent que les élèves poursuivant des buts de maîtrise comparent également leurs performances aux autres dans le but de s'évaluer et de s'améliorer. Lorsqu'on prend en compte dans l'analyse les trois types de buts simultanément, on constate que seuls les buts de maîtrise agissent comme modérateur du BFLPE. Il est toutefois à noter qu'avoir des buts de maîtrise augmente le bénéfice d'être dans une classe faible, mais n'augmente pas l'effet négatif d'être dans une classe performante.

\section{Les traits de personnalité comme modérateurs du BFLPE}

Jonckmann, Becker, Marsh et alii (2012) ont étudié six traits de personnalité comme potentiels modérateurs du BFLPE. II s'agit respectivement du caractère névrosé, extraverti, ouvert, consciencieux, agréable et narcissique de la personne. Chacun de ces traits de caractère a été mesuré par questionnaire au travers de plusieurs items avec échelle de Likert. Deux traits ressortent comme étant des modérateurs du BFLPE : la névrose et le narcissisme. Ainsi, les personnes émotionnellement instables, qui ont un haut niveau d'anxiété et qui se tracassent excessivement ont tendance à être plus sensibles au BFLPE : à compétences égales, les performances moyennes de l'école fréquentée ont un effet plus négatif sur le concept de soi académique de ces personnes. Le deuxième trait de caractère qui modère le BFLPE est le narcissisme. À compétences égales, les personnes qui se sentent supérieures, qui ont des attitudes auto-suffisantes, autoritaires et dominantes sont moins touchées par l'effet négatif des performances moyennes de l'école qu'elles fréquentent.

\section{Pratiques pédagogiques et caractéristiques de la classe comme modérateurs du BFLPE}

Marsh et Craven (2002) ont fait I'hypothèse que certaines pratiques pédagogiques de classe pouvaient modérer le BFLPE. En effet, les comparaisons mises à l'œuvre dans le BFLPE pourraient être accentuées dans un environnement très compétitif où les élèves sont incités à comparer leurs performances aux autres. À l'inverse, mettre en place des stratégies telles que donner des feed-back basés sur la personne et ses progrès plutôt qu'en comparaison aux autres ou encore valoriser chaque élève pour ce qu'il a fait même si l'élève reste plus faible que les autres pourrait réduire les comparaisons et donc le BFLPE. Marsh et Craven (2002) en sont toutefois restés aux spéculations et n'ont pas réalisé de recherche afin de confirmer ces hypothèses. En reprenant ces dernières, Lüdtke, Köller, Marsh et alii (2005) ont testé dans quelle mesure le point de référence qu'utilisent les enseignants pour formuler leurs feed-back influence le BFLPE. Par "point de référence», ils entendent le fait que l'enseignant compare les résultats de l'élève à ceux des autres (feed-back normatif) ou, au contraire, qu'il évalue les résultats de l'élève en référence à ses performances antérieures (feed-back individualisé). Leur hypothèse est que les élèves des classes où les enseignants utilisent des feed-back individualisés, c'est-à-dire sollicitant moins de comparaisons sociales, sont moins affectés par le BFLPE.

occupation de l'élève de démontrer sa compétence, alors que les buts de performance-évitement, sous-tendus par un motif de défense de soi, se traduisent par un souci d'éviter de démontrer son incompétence» (Galand \& Bourgeois, 2006, p. 71). 
Dans cette étude, le type de feed-back utilisé par l'enseignant est appréhendé des deux manières : grâce à un questionnaire soumis aux élèves et permettant de mesurer leur perception du type de feed-back utilisé par l'enseignant (exemple : «Si je fais des efforts pour accomplir mon travail, l'enseignant me félicite même si d'autres ont fait mieux que moi ») et grâce aux enregistrements vidéo et observations de leçons de mathématiques. L'analyse des données allemandes permet de constater que si l'utilisation de feed-back plus individualisés est positivement associée au concept de soi en mathématiques, celle-ci ne modère en aucun cas le BFLPE. L'effet négatif des performances moyennes de la classe est autant présent dans les classes où l'enseignant fait des feed-back individualisés que dans celles où les feed-back sont normatifs, ce qui démontre encore une fois toute la puissance du BFLPE.

Thijs, Verkuyten et Helmond (2010) ont fait I'hypothèse que la taille de la classe pouvait également jouer un effet modérateur sur le BFLPE, pour deux motifs principaux. Premièrement, selon ces auteurs, pour que l'effet négatif des performances moyennes de la classe apparaisse, il faut que les élèves aient des informations précises sur les performances de leurs pairs, ce qui est probablement plus difficile dans de grandes classes. Les élèves fréquentant de grandes classes seraient ainsi moins affectés par les performances moyennes de leurs pairs. Deuxièmement, les grandes classes peuvent apporter davantage d'opportunités de comparaisons ascendantes libres qui sont associées à un meilleur concept de soi, et donc modérer le BFLPE. Les auteurs font donc l'hypothèse d'un BFLPE moins prononcé dans les grandes classes. Les résultats de l'étude menée dans soixante-dix-neuf classes de fin de primaire aux Pays-Bas montrent toutefois qu'il n'en est rien : la taille de la classe n'entre pas en interaction avec le BFLPE.

En conclusion, il apparaît que les études peinent à mettre en évidence des variables, tant au niveau des caractéristiques de l'élève que des pratiques pédagogiques, qui jouent un rôle clair de modérateur du BFLPE. Seuls l'anxiété et le sexe ressortent comme pouvant moduler ce phénomène, mais de façon marginale. Ce résultat ne remet toutefois pas en cause le caractère généralisable du BFLPE puisque dans tous les cas nous sommes bien en présence d'un effet négatif des performances moyennes de l'école sur le concept de soi académique. Il est à noter que ces effets d'interaction ont toujours été étudiés de manière isolée. Il pourrait être intéressant d'envisager de possibles interactions entre ces variables, par exemple entre le niveau d'aptitude et le degré d'anxiété, entre le sexe et le degré de compétitivité en classe...

\section{Persistant...}

Un certain nombre d'études ont permis d'étudier le BFLPE au départ de données longitudinales (Marsh, 1991 ; Marsh, Trautwein, Lüdtke et al., 2007; Marsh \& O'Mara, 2010). L'objectif de celles-ci était de mettre en évidence le caractère persistant ou non du BFLPE.

En 1991, Marsh est le premier à tester le BFLPE au départ d'une base de données longitudinale. L'étude High School and Beyond menée aux États-Unis suit une cohorte de 14825 élèves à deux moments du secondaire (grades 10 et 12) et deux ans après l'obtention de leur diplôme. Marsh (1991) constate qu'à performances individuelles égales, il existe bien un effet négatif des performances moyennes de l'école fréquentée mesurée au grade 10 sur le concept de soi académique aux grades 10 et 12 . Le concept de soi académique n'a par contre pas été mesuré deux ans après la sortie de l'enseignement secondaire. À ce moment, seuls les aspirations et le fait de fréquenter l'enseignement supérieur ont été mesurés. Ils constatent également un effet négatif des performances moyennes de l'école sur ces variables.

Marsh, Köller et Baumert (2001) ont récolté des données concernant le concept de soi en mathématiques d'élèves allemands du grade 7 en début, milieu et fin d'année scolaire 19911992. Ils constatent que les performances moyennes de la classe, mesurées en début d'année, ont une influence négative sur le concept de soi aux différents moments de l'année scolaire. Le BFLPE agit donc bien tout au long de l'année scolaire. 
Marsh, Trautwein, Lüdtke et alii (2007) analysent quant à eux des données longitudinales récoltées en Allemagne à trois moments différents : en fin de secondaire, deux ans et quatre ans après l'obtention du diplôme de secondaire. L'objectif premier de cette étude est d'évaluer les effets de la performance moyenne de l'école fréquentée sur le concept de soi en mathématiques en fin de secondaire ainsi que deux et quatre ans après. Les auteurs constatent qu'à performances de l'élève égales, les performances moyennes de l'école fréquentée en fin de secondaire ont un effet négatif sur le concept de soi en mathématiques en fin de secondaire, mais également deux ans après, et cela même après avoir pris en compte le concept de soi académique en mathématiques en fin de secondaire. À l'inverse, ils observent que, sous contrôle des performances individuelles et du concept de soi en mathématiques en fin de secondaire, les performances moyennes de l'école fréquentée en secondaire n'ont pas d'influence sur le concept de soi en mathématiques quatre ans après. Autrement dit, les performances moyennes de l'établissement n'ont pas d'effet négatif supplémentaire sur le concept de soi en mathématiques quatre ans après le secondaire, ce dernier s'expliquant par le concept de soi en mathématiques en fin de secondaire.

Enfin, Marsh et O'Mara (2010) partent de données longitudinales récoltées en cinq vagues (grade 10, grade 11, grade 12, un an après la fin du secondaire et cinq ans après la fin du secondaire) aux États-Unis entre 1966 et 1974. Le concept de soi académique a uniquement été mesuré aux grades 10 et 11 ainsi qu'un an après la fin du secondaire. Dans le souci de tenir compte du caractère longitudinal et hiérarchisé de leurs données, ces auteurs ont mené des analyses complexes. Retenons de celles-ci que les performances moyennes de l'établissement fréquenté au secondaire ont une influence négative sur le concept de soi académique au grade 10 et un an après la fin de secondaire, mais pas au grade 11 .

Les résultats de ces différentes études tendent donc à démontrer le caractère persistant du BFLPE. Si les performances moyennes de l'établissement fréquenté ont un impact négatif sur le concept de soi académique mesuré au même moment, cet effet perdure également et influence également le concept de soi mesuré plus tard.

\section{Étendu à d'autres variables}

Très vite, Marsh (1991) a envisagé la possibilité que le BFLPE puisse agir sur d'autres dimensions que le concept de soi académique. Plus précisément, Marsh émet l'hypothèse que le concept de soi académique peut être un médiateur de l'effet des performances moyennes de l'école sur des variables telles que les aspirations scolaires et professionnelles. Il confirme cette hypothèse au départ des données longitudinales High School and Beyond Study : à performances individuelles égales, les performances moyennes de l'école fréquentée influencent négativement les aspirations professionnelles. De plus, l'effet des performances individuelles et des performances moyennes de l'école est presque entièrement médié par le concept de soi académique. En effet, à concept de soi académique équivalent, les performances de l'élève et les performances moyennes de l'école n'ont plus d'effet significatif sur les aspirations scolaires et professionnelles.

De façon similaire, Marsh et O'Mara (2010) ont observé au départ de données longitudinales un effet négatif à long terme des performances moyennes de l'école fréquentée sur les aspirations scolaires et professionnelles. Ils observent cet effet sur une durée d'environ huit ans. Ils notent également que cet effet est complètement médié par le concept de soi académique: sous contrôle du concept de soi académique, l'effet des performances moyennes de l'école sur les aspirations est proche de zéro.

Enfin, Nagengast et Marsh (2012) étudient également les aspirations professionnelles mais cette fois spécifiquement relatives à des carrières scientifiques, au départ des données PISA 2006. Ils constatent ainsi qu'à performances équivalentes, aspirer à une carrière scientifique 
est positivement lié aux performances de l'élève et négativement aux performances moyennes de l'école fréquentée et cela dans trente-et-un des cinquante-six pays étudiés. Il est toutefois à noter que ce lien a une ampleur moins importante que lorsqu'on étudie le concept de soi. Nagengast et Marh (2012) font l'hypothèse que le cadre de référence offert par les autres élèves de l'école a plus de sens lorsqu'il s'agit d'évaluer ses propres habiletés que de développer des aspirations professionnelles. Un autre résultat important de cette étude est que, sous contrôle du concept de soi en sciences, l'effet des performances moyennes de l'école sur les aspirations diminue de manière substantielle et n'est plus significatif que dans quinze pays. Ces auteurs mettent donc une fois de plus en évidence le rôle médiateur du concept de soi académique sur les aspirations.

Dupriez, Monseur, Van Campemhoudt et alii (2012) ont étudié l'impact de la composition académique de l'école fréquentée sur une variable légèrement différente : I'aspiration à réaliser des études universitaires. Ils réalisent leur étude au départ des données PISA 2003 pour vingthuit pays distincts. Contrairement à Marsh (1991), Marsh et O'Mara (2010) et Nagengast et Marsh (2012), ils n'observent toutefois pas systématiquement un effet négatif des performances moyennes de l'école sur les aspirations scolaires dans tous les pays. En effet, les auteurs constatent un effet positif dans quinze pays (Hongrie, Grèce, Corée, Japon, Autriche, Pays-Bas, Luxembourg, Slovaquie, France, Allemagne, Belgique, République tchèque, Portugal, Italie et Suisse) et identifient ceux-ci comme ayant la particularité d'organiser des filières d'enseignement de façon précoce (à l'exception de la France qui les organisent à partir de quinze ans). À l'inverse, les auteurs identifient les treize pays restants comme se caractérisant par un système éducatif davantage intégré, c'est-à-dire n'organisant pas de filières avant l'âge de quinze ans. Pour neuf de ces pays, les auteurs constatent un effet non significatif des performances moyennes de l'école sur les aspirations scolaires (Finlande, Australie, Islande, Royaume-Uni, Irlande, Norvège, Suède, Pologne et Danemark) et, pour les quatre autres, un effet négatif (Canada, Nouvelle-Zélande, États-Unis et Espagne). Pour mieux comprendre l'effet positif des performances moyennes de l'école sur les aspirations scolaires dans les pays différenciés, les auteurs reproduisent l'analyse, mais en ne gardant que les élèves qui fréquentent la filière générale. Cela permet, en effet, de neutraliser l'effet qui serait dû à une différence de curriculum en fonction de la filière fréquentée. Ils observent toutefois des résultats forts similaires: à l'exception des Pays-Bas et de la République tchèque où l'effet est devenu non significatif, dans les autres pays l'effet positif persiste. Dupriez, Monseur, Van Campenhoudt et alii (2012) expliquent ce résultat par un effet d'assimilation plus fort que l'effet de contraste dans ces pays où les filières sont précoces.

Marsh, Trautwein, Lüdtke et alii (2008) étudient également l'effet des performances moyennes de l'école fréquentée, mais sur de tout autres variables. Ils partent du principe que le concept de soi académique implique des comparaisons normatives à l'inverse d'autres variables socio-affectives qui impliquent moins de comparaisons sociales. Parmi ces variables, les auteurs étudient plus spécifiquement le sentiment $d^{\prime}$ efficacité général ${ }^{5}$, le contrôle de ses attentes $^{6}$, la persistance ${ }^{7}$ et les stratégies de contrôle ${ }^{8}$. Ils constatent ainsi que le BFLPE est systématiquement moins fort, voire inexistant, pour ces dimensions. L'effet négatif des performances moyennes est présent, mais faible dans le cas du sentiment d'efficacité général et le contrôle de ses attentes, non significatif pour la persistance et les stratégies de contrôle.

Trautwein, Lüdtke, Marsh et alii (2006) ont étudié l'effet des performances moyennes de l'établissement sur l'intérêt pour les mathématiques en raison de l'importance de cette variable 
dans l'effort, la persistance et les choix d'orientations scolaires. Ils font, d'une part, l'hypothèse d'un effet d'assimilation lié à la filière fréquentée en raison du message qui peut être porté à I'attention des élèves des filières plus faibles : il serait moins attendu d'eux qu'ils s'engagent et soient performants dans les matières plus scolaires telles que les mathématiques. D'autre part, ils postulent un effet négatif des performances moyennes de l'école, comme c'est le cas pour le concept de soi, puisque ces deux variables sont liées. Ils notent ainsi qu'à compétences égales, les performances moyennes de l'école fréquentée influencent négativement l'intérêt en mathématiques. Toutefois, ce lien disparaît quand le concept de soi en mathématiques est tenu sous contrôle. Ce résultat met encore une fois en évidence l'importance du concept de soi académique dans la compréhension du fonctionnement de l'individu au niveau scolaire. Il est également à noter qu'ils n'observent pas d'effet d'assimilation.

\section{Le BFLPE : effet classe ou effet école?}

Dans l'ensemble des recherches évoquées jusqu'ici, le cadre de référence est opérationnalisé soit au travers des performances moyennes de l'école, soit au travers de celles de la classe. Dans la plupart des cas, les recherches étudiant l'effet des performances au niveau de l'école sont celles qui utilisent les bases de données internationales telles que PISA. Dans ce cas, travailler au niveau école est davantage lié aux contraintes des bases de données disponibles qu'à un véritable choix. Néanmoins, peu d'études se sont interrogées sur l'intérêt d'adopter l'un ou l'autre niveau de données. Pourtant, les résultats d'une recherche en laboratoire menée en psychologie sociale (Zell \& Alicke, 2009) montrent que les individus ont davantage tendance à utiliser les informations les plus locales dans les processus de comparaisons sociales, c'est ce qu'on appelle l'effet de la dominance locale.

Sur cette base, Marsh, Kuyper, Morin et alii (2014) ont fait l'hypothèse que, dans le cas du $B F L P E$, la composition académique de la classe (qui est un cadre de référence plus proche de l'élève) serait un meilleur prédicteur du concept de soi académique que la composition académique de l'école. Plus précisément, ils postulent qu'en cas d'un effet de dominance locale complet, l'effet de la composition académique de l'école devrait être nul sous contrôle de la performance moyenne de la classe. Au départ d'une large base de données composée de 15356 élèves néerlandais du grade 9 issus de 651 classes de 65 écoles, Marsh, Kuyper, Morin et alii (2014) ont testé cette hypothèse à l'aide d'un modèle à trois niveaux (élèves-classesécoles). En accord avec leur hypothèse, les auteurs constatent que l'effet négatif des performances moyennes de l'école disparaît complètement sous contrôle des performances moyennes de la classe. Ce constat suggère que l'effet du contexte de l'école observé jusqu'ici est en fait le reflet cumulé des effets classe, les écoles performantes étant plus souvent constituées de classes elles-mêmes performantes.

Liem, Marsh, Martin et alii (2013) ont également étudié l'effet de dominance locale dans le cas du BFLPE. Ils étudient ainsi les effets des performances moyennes de l'école, de la filière et de la classe au départ de données singapouriennes. À Singapour, au sein des écoles, les élèves du secondaire sont répartis dans trois filières distinctes (très performante, moyenne, faible) en fonction de leurs résultats à un même test. En concordance avec la théorie de la dominance locale, les auteurs font l'hypothèse que si ces trois niveaux de performances moyennes ont une influence négative sur le concept de soi académique, le plus local devrait avoir un effet plus saillant que les autres. Ils confirment bien la première partie de leur hypothèse : intégrées dans les analyses de façon distincte, les performances moyennes de l'école, de la filière et de la classe ont chacune une influence négative significative sur le concept de soi tant en mathématiques qu'en anglais. Par contre, l'analyse de l'effet simultané des performances moyennes de l'école, de la filière et de la classe fait apparaître un effet de dominance locale. D'une part, dans le cas du concept de soi en mathématiques, l'effet des performances 
moyennes de l'école et de la classe devient non significatif tandis que les performances moyennes de la filière ont toujours un effet négatif significatif. D'autre part, dans le cas du concept de soi en anglais, seul l'effet des performances moyennes de l'école devient non significatif, les performances moyennes de la classe ainsi que celles de la filière conservant un effet négatif. Cette étude (Liem, Marsh, Martin et al., 2013) démontre donc la prédominance de la classe ou de la filière dans la formation du concept de soi académique.

\section{Conclusion}

La question de l'effet des performances moyennes de l'école ou de la classe fréquentée sur le concept de soi académique a été étudiée à maintes reprises ces dernières années. Alors que les élèves fréquentant les écoles, les filières ou les classes les plus performantes en tirent d'incontestables bénéfices sur le plan de leurs apprentissages et acquis scolaires, les avantages apparaissent nettement moindres, voire se changent en désavantages, sur le plan de la motivation et plus particulièrement du concept de soi académique. Cet effet négatif des performances moyennes de l'établissement fréquenté sur le concept de soi de l'élève a été repris sous le terme de BFLPE. Une vue d'ensemble des études sur le BFLPE met en évidence le caractère universel, généralisable et pérenne de celui-ci. Ce résultat cache toutefois toute la complexité de ce phénomène. Les différentes études prises en compte dans cette note de synthèse amènent en effet à pointer une série d'éléments importants lorsqu'on étudie en profondeur le BFLPE. Si toutes ces études prennent en compte des tests standardisés et utilisent des analyses multiniveaux, elles divergent quant à la nature des bases de données et au niveau d'analyse (classe, filière, école). Ces deux critères de recherche apparaissent pourtant comme cruciaux.

Si I'on souhaite comprendre en profondeur le BFLPE et la manière dont effet de contraste et effet d'assimilation s'imbriquent, I'utilisation de données nationales semble plus opportune. Alors que les données internationales ont permis de mettre en évidence l'universalité du BFLPE, travailler avec de telles données soulève un certain nombre de limites : les performances moyennes de l'école sont agrégées sur base d'un échantillon d'élèves de l'école et les données ne permettent pas d'analyser les choses au niveau classe. Les données nationales permettent quant à elles une meilleure description du phénomène grâce à une connaissance plus fine du système éducatif. En effet, s'il apparaît dans la plupart des études que le BFLPE peut être considéré comme la résultante d'un effet de contraste supérieur à l'effet d'assimilation, des études menées en Allemagne, où le système scolaire est très différencié, permettent de mettre en évidence que la forme sous laquelle les filières d'enseignement sont organisées peut changer les choses. Adopter un point de vue national permet ainsi d'appréhender toute la complexité du système éducatif : les filières correspondent-elles aux écoles ou sont-elles organisées au sein de celles-ci? Ces filières au sein des écoles correspondent-elles à des classes? Ces questions apparaissent comme centrales pour comprendre en profondeur le BFLPE. En Allemagne, deux types de filières sont organisées : les filières qui correspondent aux écoles (Gymnasium, Realschule et Hauptschule) et les classes de niveau organisées au sein des écoles selon les aptitudes des élèves en mathématiques, langues étrangères et allemand. Les recherches semblent mettre en évidence que lorsqu'on considère les filières au niveau de l'école, un effet de contraste lié aux performances moyennes de la classe est systématiquement observé alors que ce n'est pas le cas en ce qui concerne l'effet d'assimilation lié à la filière fréquentée. En effet, Trautwein, Lüdtke, Marsh et alii (2006) ont mis en évidence un effet négatif de la filière fréquentée quand celle-ci correspond à l'école : les élèves qui fréquentent la filière la plus "prestigieuse» ont un concept de soi académique inférieur aux autres. Par contre, lorsqu'il s'agit des classes de niveau organisées au sein des écoles, on observe qu'un effet positif de la filière fréquentée s'additionne toujours à l'effet de contraste. Ainsi, dans le cas allemand, l'effet 
d'assimilation (positif pour les meilleurs élèves et négatif pour les moins bons) que l'on peut retirer de la fréquentation d'une filière plus ou moins exigeante ne percole que quand les filières s'organisent à l'intérieur de l'école et correspondent à des classes de niveau - ce qui est aisé à comprendre : les élèves peuvent alors se comparer «en local» aux élèves d'autres filières.

Utiliser le prestige perçu de l'école ou de la classe apparaît également comme un puissant moyen de distinguer effet de contraste et effet d'assimilation. Les deux études réalisées dans cette optique (Marsh, Kong \& Hau, 2000; Trautwein, Lüdtke, Marsh et al., 2009) aboutissent à des résultats similaires : un effet négatif des performances moyennes de l'école ou de la classe coexiste avec un effet positif du prestige perçu de cette école ou classe. Dans les systèmes éducatifs différenciés, la filière apparaissant comme centrale dans la compréhension des effets de contraste et d'assimilation, il pourrait être intéressant d'envisager le prestige perçu de la filière fréquentée, en particulier dans les cas où la filière d'enseignement ne correspond ni à l'école ni à la classe.

Par ailleurs, cette approche plus fouillée des filières d'enseignement permettrait sans doute de mieux comprendre les résultats surprenants avancés par Dupriez, Monseur, Van Campenhoudt et alii (2012). À l'inverse de ce qui est observé pour le concept de soi académique, à savoir que le BFLPE est plus fort dans les systèmes éducatifs différenciés, ces auteurs n'observent pas de BFLPE pour les aspirations scolaires dans ces mêmes systèmes éducatifs. Ils y constatent même un effet positif des performances moyennes de l'école. Les auteurs font I'hypothèse que cet effet positif serait lié à la filière fréquentée. Il serait donc intéressant d'étudier dans quelle mesure cet effet positif correspond à un effet d'assimilation lié au sentiment des élèves de fréquenter une filière d'enseignement plus prestigieuse et préparant en principe aux études supérieures. Existe-t-il néanmoins dans ce cas de figure un effet de contraste qui serait alors plus faible que l'effet d'assimilation? Cette étude devrait plus spécifiquement porter sur des données nationales pour lesquelles une bonne connaissance du système est nécessaire afin de mettre en lumière ce qui se passe réellement sous cet effet.

Une deuxième dimension qui distingue les études réalisées sur le BFLPE est le niveau d'analyse pris en compte. Certaines études envisagent les performances moyennes au niveau de l'école tandis que d'autres les envisagent au niveau de la classe. Il apparaît que les mécanismes de comparaison sociale - effet de contraste (BFLPE) ou d'assimilation (prestige perçu) opèrent surtout au niveau le plus local (dominance locale) : pour évaluer ses capacités en mathématiques par exemple, l'élève se compare d'abord aux élèves de sa classe plutôt qu'à ceux de son école. En effet, Marsh, Kuyper, Morin et alii (2014) ainsi que Liem, Marsh, Martin et alii (2013) ont pu mettre en évidence que lorsque les performances moyennes de la classe ou de la filière sont prises en compte, l'effet des performances moyennes de l'école n'est plus significatif. Dans la mesure du possible, l'étude du BFLPE devrait donc être réalisée au départ de données «classe » ou, si elle est réalisée avec des données «école», envisager la possibilité que le BFLPE soit sous-estimé.

L'analyse de données internationales et nationales a également permis de mettre en évidence qu'à performances individuelles égales de l'élève, l'impact négatif de performances moyennes élevées de l'école sur le concept de soi s'observe davantage dans les systèmes différenciés que dans les systèmes peu différenciés. Cette caractéristique du système éducatif dans lequel est étudié le BFLPE est donc à prendre en considération dans l'analyse des résultats.

Deux autres éléments semblent devoir être pointés à l'issue de cette note de synthèse. Tout d'abord, si l'on souhaite vraiment isoler le BFLPE, il est nécessaire de prendre en compte les notes données par l'enseignant, puisque le caractère normatif de celles-ci explique en partie l'effet négatif des performances moyennes de l'établissement (Marsh, 1987; Trautwein, Lüdtke, Marsh et al., 2006; Marsh, Trautwein, Lüdtke et al., 2007; Preckel \& Brüll, 2010). En effet, 
les élèves s'appuient davantage, pour définir leur concept de soi académique, sur les notes scolaires et ce qu'elles reflètent (attentes, exigences plus ou moins élevées des enseignants) que sur leurs capacités objectivées par la passation d'un test standardisé. Enfin, s'il est envisagé d'étudier le BFLPE à l'égard d'autres variables que le concept de soi académique, il sera alors nécessaire de prendre en compte que ce dernier joue un rôle de médiateur incontestable par rapport à d'autres variables telles que les aspirations ou encore l'intérêt pour les mathématiques.

À un niveau plus pratique, quelles implications retenir de cette note? Au vu de l'existence du BFLPE dans de nombreux contextes, les parents ayant la possibilité de choisir un établissement pour leur enfant, en particulier dans les systèmes fortement différenciés où les contrastes entre écoles sont marqués, devraient connaître et prendre en compte le possible effet négatif sur le plan socio-affectif de fréquenter une école très performante. Vouloir à tout prix le «meilleur » établissement, la filière la plus prestigieuse pour son enfant n'est pas toujours et nécessairement le meilleur choix, et peut même se révéler délétère si l'élève est particulièrement sensible aux comparaisons sociales (enfant anxieux, filles dans les matières scientifiques). Pour un élève aux aptitudes relativement moyennes, il s'agit donc de mettre en balance les indéniables avantages, sur le strict plan des acquis scolaires, de fréquenter une école ou une classe plus performante et les possibles désavantages que ceci peut entraîner sur le plan socioaffectif. Dans cet arbitrage, il faudra aussi prendre en compte le fait que la mesure du concept de soi sur laquelle s'appuient les études relatives au BFLPE, en dépit de ses qualités psychométriques, n'est qu'une mesure auto-rapportée, susceptible d'un certain nombre de biais ou de styles de réponses eux-mêmes liés à certaines caractéristiques des élèves ou des groupes (Lafontaine, 2017; Van de Vijver \& Tanzer, 2004). À un niveau plus politique, au vu des résultats des recherches évoquées dans cette note de synthèse, on ne peut que plaider en faveur de plus de mixité au sein des écoles afin d'atténuer les effets liés au contexte scolaire qui s'additionnent aux effets des caractéristiques individuelles.

En conclusion, s'il ne fait aucun doute que la composition académique de l'école, de la filière ou de la classe fréquentée joue un rôle fondamental et généralisé dans le développement socio-affectif du jeune, la compréhension de cet effet reste complexe et, comme le montrent certaines des études les plus récentes, étroitement dépendante de caractéristiques spécifiques du contexte. Mener des études ayant pour objectif d'éclairer davantage les mécanismes fins sous-jacents au BFLPE dans une variété de systèmes éducatifs, en prenant notamment en compte la manière dont les niveaux classe, filière et s'y emboîtent, apparaît comme primordial.

Virginie Dupont Université de Liège, service d'Analyse des Systèmes et des Pratiques d'enseignement virginie.dupont@ulg.ac.be

Dominique Lafontaine Université de Liège, service d'Analyse des Systèmes et des Pratiques d'enseignement dlafontaine@ulg.ac.be 
BLANTON H., BUUNK B. P., GIBBONS F. X. \& KUYPER H. (1999). «When better-than-others compare upward: Choice of comparison and comparative evaluation as independent predictors of academic performance ». Journal of Personality end Social Psychology, n76, p. 420-430.

CASLYN R. J. \& KENNY D. A. (1977). «Self-concept of ability and perceived evaluation of others: Cause or effect of academic achievement? » Journal of Educational Psychology, vol. 69, n², p. 136-145.

CHANAL J. P. \& SARRAZIN P. G. (2007). «Big-fish-little-pond effect versus positive effect of upward comparisons in the classroom: how does one reconcile contradictory results?» Revue internationale de psychologie sociale, $\mathrm{n}^{\circ} 20$, p.69-86.

DUPRIEZ V., MONSEUR C., VAN CAMPENHOUDT M. \& LAFONTAINE D. (2012). «Social inequalities of postsecondary educational aspirations: influence of social background, school composition and institutional context». European Educational Research Journal, vol. 11, n 4, p. 504-519.

DURU-BELLAT M. (2003). «Les apprentissages des élèves dans leur contexte : Les effets de la composition de l'environnement scolaire». Carrefours de l'Éducation, n¹6, p. 183-206.

DURU-BELLAT M., DANNER M., LE BASTARD-LANDRIER S. \& PIQUÉE C. (2004). «Les effets de la composition scolaire et sociale du public d'élèves sur leur réussite et leurs attitudes : évaluation externe et explorations qualitatives ». Les Cahiers de I'IREDU, vol. 65, p. 144-155.

FELOUZIS G. (2003). «La ségrégation ethnique au collège et ses conséquences». Revue française de sociologie, vol.44, p. 413-447.

GALAND B. \& BOURGEOIS E. (2006). (Se) Motiver à apprendre. Paris : PUF.

GAVRAY C. \& ADRIAENSSENS A. (2010). Une fille = un garçon ? Identifier les inégalités de genre à l'école pour mieux les combattre. Paris : L'Harmattan.

HANSFORD B. C. \& HATTIE J. A. (1982). «The relationship between self and achievement/performance measures». Review of Educational Research, $\mathrm{n}^{\circ}$ 52, p. 123-142.

HUGUET P., DUMAS F., MONTEIL J.-M. \& GENESTOUX N. (2001). «Social comparison choices in the classroom: Further evidence for students' upward comparison tendency and its beneficial impact on performance ». European Journal of Social Psychology, n`31, p. 557-578.

HUGUET P., DUMAS F., MARSH H. W., RÉGNER I., WHEELER L., SULS J., SEATON M. \& NEZLEK J. (2009). "Clarifying the role of social comparison in the Big-Fish-Little-Pond effect (BFLPE): an integrative study ». American Psychology Association, vol. 97, n¹, p. 156-170.

JONKMANN K., BECKER M., MARSH H. W., LÜDTKE O. \& TRAUTWEIN U. (2012). «Personality traits moderate the Big-Fish-Little-Pond effect of academic self-concept». Learning and Individual Differences, $\mathrm{n}^{\circ} 22$, p.736-746.

LAFONTAINE D. (2017). «Évaluations à large échelle : prendre la juste mesure des effets de contexte». In P. Detroz, M. Crahay \& A. Fagnant, L'évaluation à la lumière des contextes et des disciplines. Bruxelles: De Boeck.

LEYENS J.-P. \& YZERBYT V. (1997). Psychologie sociale. Sprimont : Mardaga.

LIEM G. A. D., MARSH H. W., MARTIN A. J., MCINERNEY D. M. \& YEUNG A. S. (2013). «The Big-Fish-LittlePond effect and a national policy of within-School ability streaming: Alternative Frames of Reference». American Educational Research Journal, vol.50, n², p.326-370.

LÜDTKE O., KÖLLER O., MARSH H. W. \& TRAUTWEIN U. (2005). «Teacher frame of reference and the bigfish-little-pond effect». Contemporary Educational Psychology, n³0, p.263-285.

MARSH H. W. (1987). «The big-fish-little-pond effect on academic self-concept». Journal of Educational Psychology, vol.79, n³, p.280-295.

MARSH H. W. (1991). «Failure of high-ability high schools to deliver academic benefits commensurate with their student's ability levels». American Educational Research Journal, vol.28, n², p. 445-480.

MARSH H. W., ABDULJABBAR A. S., MORIN A.J. S., PARKER P., ABDELFATTAH F., NAGENGAST B. \& ABU-HILAL M. M. (2015). «The Big-fish-little-pond effect: Generalizability of social comparison processes over two age cohorts from western, Asian, and middle eastern Islamic countries ». Journal of Educational Psychology, vol. 107, $\mathrm{n}^{\circ} 1$, p. 258-271.

MARSH H. W., CHESSOR D., CRAVEN R. \& ROCHE L. (1995). «The effects of gifted and talented programs on academic self-concept: The big fish strikes again ». American Educational Research Journal, vol.32, $n^{\circ} 2$, p. 285-319. 
MARSH H. W. \& CRAVEN R. (2002). «The pivotal role of frames of reference in academic self-concept formation: The big-fish-little-pond effect ». In F. Pajares \& T. Urdan, Adolescences and education (Vol. II). Greenwich : Éd. Information Age, p. 83-123.

MARSH H. W. \& HAU K.-T. (2003). «Big-fish-little-pond effect on academic self-concept. A cross-cultural (26-Country) test of the negative effects of academically selective schools ». American Psychologist, vol.58, n5, p.364-376.

MARSH H. W., KÖLLER O. \& BAUMERT J. (2001). «Reunification of East and West German school systems: Longitudinal multilevel modeling study of the big-fish-little-pond effect on academic self-concept ». American Educational Research Journal, n³8, p.321-350.

MARSH H. W., KONG C.-K. \& HAU K.-T. (2000). «Longitudinal multilevel models of the big-fish-little-pond effect on academic self-concept: Counterbalancing contrast and reflected-glory effects in Hong Kong schools». Journal of Personality \& Social Psychology, n78, p. 337-349.

MARSH H. W., KUYPER H., MORIN A.J. S. \& PARKERP. D. (2014). «Big-fish-little-pond social comparison and local dominance effects: Integrating new statistical models, methodology, design, theory and substantive implications». Learning and Instruction, $\mathrm{n}^{\circ} 33$, p.50-66.

MARSH H. W. \& O'MARA A. J. (2010). «Long-term total negative effects of school-average ability on diverse educational outcomes. Direct and indirect effects of the Big-Fish-Little-Pond effect». Zeitschrift für Pädagogische Psychologie, vol.24, n¹, p.51-72.

MARSH H. W. \& PARKER J. W. (1984). «Determinants of students self-concept: Is it better to be a relatively large fish in a small pond even if you don't learn to swim well? » Journal of Personality and Social Psychology, vol. $47, \mathrm{n}^{\circ} 1, \mathrm{p} .213-231$.

MARSH H.W., SEATON M., TRAUTWEIN U., LÜDTKE O., HAU K.T., O'MARA A.J. \& CRAVEN R.G., (2008). «The Big-Fish-Little-Pond effect stands up to critical scrutiny: implications for theory, methodology, and future research ». Educational Psychology Review, n²0, p. 319-350.

MARSH H. W., TRAUTWEIN U., LÜDTKE O., BAUMERT J. \& KÖLLER O. (2007). «The Big-Fish-Little-Pond effect: Persistent negative effects of selective high schools on self-concept after graduation ». American Educational Research Journal, vol.44, n³, p. 631-669.

MARSH H. W., TRAUTWEIN U., LÜDTKE O. \& KÖLLER O. (2008). «Social Comparison and Big-Fish-LittlePond Effects on Self-concept and other self-belief constructs: Role of generalized and specific others ». Journal of Educational Psychology, vol.100, n³, p.510-524.

MONSEUR C. \& LAFONTAINE D. (2009). «L'organisation des systèmes éducatifs : Quel impact sur l'efficacité et l'équité? ». In X. Dumay \& V. Dupriez, L'efficacité dans l'enseignement. Promesses et zones d'ombres. Bruxelles : De Boeck, p.141-136.

MÜLLER C. M. \& HOFMANN V. (2014). «Does being assigned to a low track negatively affect psychological adjustment. A longitudinal study in the first year of secondary school ». School Effectiveness and School improvement, vol.27, $\mathrm{n}^{\circ} 2$, p. 95-115.

NAGENGAST B. \& MARSH H. W. (2011). «The negative effect of school-average ability on self-concept in the UK, the UK countries and the world: the Big-Fish-Little-Pond-Effect for PISA 2006 ». Educational Psychology, vol.31, n5, p. 629-656.

NAGENGAST B. \& MARSH H. W. (2012). «Big fish little ponds aspire more: mediation and cross-cultural generalizability of school-average ability effects on self-concept and career aspirations in sciences ». Journal of Educational Psychology, vol. 104, n 4, p. 1033-1053.

NAKHILI N. (2005). «Impact du context scolaire dans l'élaboration des choix d'études supérieures des élèves de terminale». Éducation et Formations, vol.72, p.155-167.

PIETSCH J., WALKER R. \& CHAPMAN E. (2003). «The relationship among self-concept, self-efficacy, and performance in mathematics during secondary school ». Journal of Educational Psychology, vol. 95, $\mathrm{n}^{\circ} 3$, p. 589-603.

PLIENINGER H. \& DICKHÄUSER O. (2015). «The female fish is more responsive: gender moderates the BFLPE in the domain of science». Educational Psychology: An International Journal of Experimental Educational Psychology, vol.35, n², p.213-227.

PRECKEL F. \& BRÜLL M. (2010). «The benefit of being a big fish in a big pond: Contrast and assimilation effects on academic self-concept ». Learning and Individual Differences, $n^{\circ} 20$, p. 522-531.

SEATON M., MARSH H. W., DUMAS F., HUGUET P., MONTEIL J.-M., RÉGNER I., BLANTON H., BUUNKA. P., GIBBONSF. X., KUYPER H., SULS J. \& WHEELER L. (2008). «In search of the big fish: Investigating the coexistence of the big-fish-little-pond effect with the positive effect of upward comparisons ». British Journal of Social Psychology, n²47, p.73-103. 
SEATON M., MARSH H. W. \& CRAVEN R. G. (2009). «Earning its place as a pan-human theory: Universality of the Big-Fish-Little-Pond effect across 41 culturally and economically diverse countries ». Journal of Educational Psychology, vol.101, n², p. 403-419.

SEATON M., MARSH H. W. \& CRAVEN R. G. (2010). «Big-Fish-Little-Pond effect : Generalizability and Moderation-Two sides of the same coin ». American Educational Research Journal, vol.47, n², p. 390-433.

SEATON M., MARSH H.W., YEUNG A.S. \& CRAVEN R. (2011). «The big fish down under: examining moderators of the "big-fish-little-pond" effect for Australia's high achievers». Australian Journal of Education, vol.55, n², p. 93-114.

SKAALVIK E. M. \& SKAALVIK S. (2002). «Internal and external frames of reference for academic selfconcept». Educational Psychologist, n³7, p. 233-244.

THIJS J., VERKUYTEN M. \& HELMOND P. (2010). «A further examination of the Big-Fish-Little-Pond effect: Perceiver position in class, class size, and gender comparisons». Sociology of Education, vol. $83, n^{\circ} 4$, p.333-345.

TRAUTWEIN U., LÜDTKE O., MARSH H. W., KÖLLER O. \& BAUMERT J. (2006). «Tracking, grading, and student motivation: Using group composition and status to predict self-concept and interest in NinthGrade Mathematics». Journal of Educational Psychology, vol. 98, n²4, p.788-806.

TRAUTWEIN U., LÜDTKE O., MARSH H. W. \& NAGY G. (2009). «Within-School Social Comparison: How students perceive the standing of their class predicts academic self-concept ». Journal of Educational Psychology, vol.101, nº 4, p. 853-866.

VAN DE GAER E., GRISAY A., SCHULZ W. \& GEBHARDT E. (2012). «The reference group effect: An explanation relationship between academic achievement and self-confidence across countries ». Journal of Cross-Cultural Psychology, vol.43, n 8, p. 1205-1228.

VAN DE VIJVER F.J. R. \& TANZERN. K. (2004). «Bias and equivalence in cross-cultural assessment: An overview ». Revue européenne de psychologie appliquée/European Review of Applied Psychology, $\mathrm{n}^{\circ}$ 54, p. 119-135.

WANG Z. (2015). «Examining big-fish-little-pond effects across 49 countries: a multilevel latent variable modelling approach ». Educational Psychology: An International Journal of Experimental Educational Psychology, vol. $35, \mathrm{n}^{\circ} 2$, p. 228-251.

WOUTERS S., COLPIN H., VAN DAMME J. \& VERSCHUEREN K. (2015). «Endorsing achievement goals exacerbates the big-fish-little-pond effect on academic self-concept ». Educational Psychology: An International Journal of Experimental Educational Psychology, vol.35, n², p. 252-270.

ZELL E. \& ALICKE M. D. (2009). «Contextual neglect, self-evaluation, and the frog-pond effect». Journal of Personality and Social Psychology, vol. 97, n³, p. 467-482. 


\section{Annexe 1}

\begin{tabular}{|c|c|c|}
\hline & Données internationales & Données nationales \\
\hline 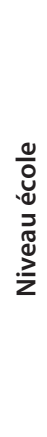 & $\begin{array}{l}\text { - Marsh \& Hau (2003) } \\
\text { - Seaton, Marsh \& Craven (2009) } \\
\text { - Seaton, Marsh \& Craven (2010) } \\
\text { - Nagengast \& Marsh (2011) } \\
\text { - Nagengast \& Marsh (2012) } \\
\text { - Van de Gaer, Grisay, Schulz et al. (2012) } \\
\text { - Seaton, Marsh, Dumas et al. (2008) } \\
\text { - Seaton, Marsh, Yeung et al. (2011) } \\
\text { - Pleininger \& Dickhäuser (2015) } \\
\text { - Marsh, Trautwein, Lüdtke et al. (2008) } \\
\text { - Dupriez, Monseur, Van Compenhoudt } \\
\text { et al. (2012) }\end{array}$ & $\begin{array}{l}\text { - Marsh \& Parker (1984) - Australie } \\
\text { - Marsh (1987) - Australie } \\
\text { - Marsh (1991) - États-Unis } \\
\text { - Marsh, Chessor, Craven et al. (1995) - } \\
\text { Australie } \\
\text { - Marsh, Kong \& Hau (2000) - Hong-Kong } \\
\text { - Marsh, Trautwein, Lüdtke et al. (2007) } \\
\text { - Allemagne } \\
\text { - Trautwein, Lüdtke, Marsh et al. (2009) - } \\
\text { Allemagne } \\
\text { - Jonckmann, Becker, Marsh et al. (2012) } \\
\text { - Allemagne } \\
\text { - Marsh \& O’Mara (2010) - Allemagne }\end{array}$ \\
\hline 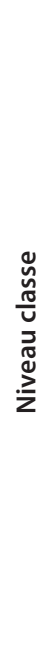 & $\begin{array}{l}\text { - Marsh, Abduljabbar, Morin et al. (2015) } \\
\text { - Wang (2015) } \\
\text { - Lüdtke, Köller, Marsh et al. (2005) (exten- } \\
\text { sion TIMSS) }\end{array}$ & $\begin{array}{l}\text { - Marsh, Köller \& Baumert (2001) - } \\
\text { Allemagne } \\
\text { - Trautwein, Lüdtke, Marsh et al. (2006) } \\
\text { (extension PISA Allemagne) } \\
\text { - Preckel \& Brüll (2010) - Allemagne } \\
\text { - Trautwein, Lüdtke, Marsh et al. (2009) - } \\
\text { Allemagne } \\
\text { - Seaton, Marsh, Dumas et al. (2008) - Pays- } \\
\text { Bas et France } \\
\text { - Thijs, Verkuyten \& Helmond (2010) - Pays- } \\
\text { Bas } \\
\text { - Wouters, Colpin, Van Damme et al. (2015) } \\
\text { - Flandre (Belgique) } \\
\text { - Huguet, Dumas, Marsh, et al. (2009) - } \\
\text { France } \\
\text { - Liem, Marsh, Martin et al. (2013) - } \\
\text { Singapour } \\
\text { - Marsh, Trautwein, Lüdtke et al. (2008) } \\
\text { - Allemagne } \\
\text { - Chanal \& Sarrazin (2007) - France }\end{array}$ \\
\hline 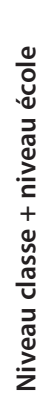 & & $\begin{array}{l}\text { - Marsh, Kuyper, Morin et al. (2014) - } \\
\text { Allemagne }\end{array}$ \\
\hline
\end{tabular}

